\title{
Chlamydia trachomatis Mouse Pneumonitis Lung Infection in IL-18 and IL-12 Knockout Mice: IL-12 Is Dominant over IL-18 for Protective Immunity
}

\author{
Hang Lu, ${ }^{1}$ Xi Yang, ${ }^{2}$ Kiyoshi Takeda, ${ }^{3}$ Dongji Zhang, ${ }^{2}$ Yijun Fan, ${ }^{2}$ Ma Luo, ${ }^{2}$ \\ Caixia Shen, ${ }^{1}$ Shuhe Wang, ${ }^{2}$ Shizuo Akira, ${ }^{3}$ and Robert C. Brunham ${ }^{1}$ \\ ${ }^{1}$ University of British Columbia, Centre for Disease Control, Vancouver, British \\ Columbia, Canada \\ ${ }^{2}$ Department of Medical Microbiology, University of Manitoba, Winnipeg, \\ Manitoba, Canada \\ ${ }^{3}$ Department of Host Defense, Research Institute for Microbial Diseases, \\ Osaka University, Osaka, Japan
}

Accepted May 1, 2000

\begin{abstract}
Background: Interferon (IFN) $-\gamma$ is a key to protective immunity against a variety of intracellular bacterial infections, including Chlamydia trachomatis. Interleukin (IL)-18, a recently identified Thl cytokine, together with IL-12 is a strong stimulator for IFN- $\gamma$ production. We investigated the relative roles of IL18 and IL-12 in protective immunity to C. trachomatis mouse pneumonitis (MoPn) infection using gene knockout (KO) and wild-type (WT) mice.

Materials and Methods: Mice were intranasally infected with $C$. trachomatis MoPn and protective immunity was assessed among groups of mice by daily body weight changes, lung growth of MoPn, and histopathological appearances at day 10 postinfection. The corresponding immune responses for each group of mice at the same postinfection time point were evaluated by measuring antigen-specific antibody isotype responses and cytokine profiles.

Results: Our results showed that IL-18 deficiency had little or no influence on clearance of MoPn from the lung, although KO mice exhibited slightly more severe inflammatory reactions in lung tissues, as well as reduced systemic and local IFN- $\gamma$ production, com-
\end{abstract}

pared with WT mice. Results with IL-18 KO mice were in sharp contrast to those observed with IL-12 KO mice that showed substantially reduced clearance of MoPn from the lungs, substantial reductions of antigen-specific systemic and lung IFN- $\gamma$ production, decreased ratio of MoPn-specific immunoglobulin G (IgG) $2 \mathrm{a} / \mathrm{IgGl}$, and severe pathological changes in the lung with extensive polymorphonuclear, instead of mononuclear, cell infiltration. Exogenous IL-12 or IL18 was able to increase IFN- $\gamma$ production in IL-18 KO mice; whereas, only exogenous IL-12, but not IL-18, enhanced IFN- $\gamma$ production in IL-12 KO mice. Caspase- 1 is the key protease for activation of IL-18 precursor into the bioactive form, and caspase-1 KO mice also displayed similar bacterial clearance and body weight loss to that in WT mice at early stages of MoPn infection. This further confirmed that IL-18 was not essential for host defense against chlamydia infection. Conclusions: These results suggest that IL-12, rather than IL-18, plays the dominant role in the development of protective immunity against chlamydia lung infection, although both cytokines are involved in the in vivo regulation of IFN $-\gamma$ production.
Address correspondence and reprint requests to: Dr. Robert C Brunham, University of British Columbia Center for Disease Control, 655 West $12^{\text {th }}$ Avenue, Vancouver, British Columbia, V5Z 4R4, Canada. Phone: 604-660-1840; Fax: 604-660-6073; E-mail: robert.brunham@bccdc.hnet.bc.ca

\section{Introduction}

Chlamydia trachomatis is an obligate intracellular bacterium that causes a variety of human diseases, including sexually transmitted diseases, trachoma, and infant pneumonia. Several cytokines are known to play critical roles in host resistance or susceptibility to chlamydial infec- 
tion, including interferon (IFN)- $\gamma$, interleukin (IL)-12, and IL-10. IL-12 is a powerful factor for the generation of $\mathrm{T}$ helper-1 (Thl) cellmediated immune responses and is required for the eradication of chlamydial genital infection $(1,2)$. Administration of exogenous IL-12 early after infection significantly alleviated the severity of chlamydial pneumonia, abolished mortality, and decreased chlamydia growth in vivo (3). IFN- $\gamma$ is essential for preventing systemic dissemination and/or eliminating chronic persistence of chlamydia infection $(1,2,4,5)$. Recently, we reported that IFN- $\gamma$ knockout (KO) mice showed Th2-associated inflammation and failed to control C. trachomatis mouse pneumonitis (MoPn) infection in the lung (6). In contrast to IFN- $\gamma$ KO mice, mice with genetic deficiency of endogenous IL-10 showed accelerated pulmonary clearance of MoPn and significantly enhanced delayedtype hypersensitivity (DTH) responses, which could be inhibited by local administration of recombinant interleukin (rIL)-10 (7). Th2biased immune responses related to the overproduction of IL-10 in inbred mouse strains were asso-ciated with increased susceptibility to chlamydia infection $(8,9)$. Overall, these results suggest that Thl cells are required for chlamydia immunity and that IL-10 inhibits the priming and expansion of Thl immune responses.

IL-18 is a recently characterized cytokine that acts as a costimulatory factor for the production of IFN- $\gamma$. It has been demonstrated that IL-18 can be produced in response to bacterial and inflammatory stimuli by various cell types, including activated mononuclear cells (10), macrophages (11), keratinocytes (12), and dendritic cells (13). IL-18 is synthesized in a precursor form and converted to a biologically active form by the protease, caspase-1 $(14,15)$. IL-18 has multiple biological functions, including induction of IFN- $\gamma$ production by natural killer (NK) cells and activated Thl cells (16), enhancement of Fas ligand-mediated cytotoxicity of murine Thl cells $(17,18)$, augmentation of NK cell cytotoxicity $(16,19)$, and up-regulation of intercellular adhesion molecular (ICAM)-1 expression on epidermal cells (20). Because of its ability to induce IFN- $\gamma$ production, IL- 18 is classified in the Thl cytokine family, together with IL-2, IL-12, IL-15, and IFN- $\gamma$. The major role for IL-18 appears to be to interact synergistically with IL-12 to enhance IFN- $\gamma$ production. The combination of IL-18 and IL-12 is far more effective at inducing IFN- $\gamma$ production than either cytokine alone $(21,22)$.

Previous studies demonstrated the importance of IL-18 in host defense against microbial infection. IL-18 KO mice show significant defects in NK cell activity and impaired Thl immune response following the intravenous infection with Propionibacterium acnes or $M y$ cobacterium bovis (16). IL-18 also contributes to host resistance in mice to infection with $M y$ cobacterium (23), Leishamania major, Staphylococcus aureaus (24), Salmonella typhimurium (25), and Herpes simplex virus (26). In the present study, we investigated the role of endogenous IL-18 in mediating protection against $C$. trachomatis MoPn lung infection using IL-18 KO mice, and compared the relative importance of IL-18 to IL-12 in host defense to MoPn infection.

\section{Materials and Methods}

Mice

Eight- to twelve-week-old C57BL/6 mice were obtained from Charles River Canada (St. Constant, Quebec, Canada). Age and sex-matched homozygous IL-18 and IL-12 p40 KO mice with C57BL/6 background were previously described (24) or purchased from Jackson Laboratories (Bar Harbor, ME), respectively. Homozygous caspase-1 KO and matched heterozygous mice were kindly provided by Dr. Winnie Wong from BASF Bioresearch Inc. (Worcester, MA). Mice were housed at the University of Manitoba animal facility in filter-top cages.

\section{Chlamydia Organism and Intranasal Inoculation}

Chlamydia MoPn was grown in Hela 229 cells and elementary bodies (EBs) were purified on discontinuous density gradients of centrifugation as previously described (27). The infectivity of purified EBs was titrated by infection of Hela cell monolayers for $36 \mathrm{hr}$, followed by the fixation of cells with methanol and enumeration of inclusions. The cells having chlamydial inclusions were detected by the staining with anti-chlamydial lipopolysaccharide monoclonal antibody (LPS mAb) (ViroStat, Portland, ME) as previously described (27). For in vitro stimulation, EBs were heat inactivated at $70^{\circ} \mathrm{C}$ for $30 \mathrm{~min}$.

Mice were intranasally inoculated with $1 \times$ $10^{4}$ inclusion forming unit (IFU) of MoPn and monitored daily for body weight changes. Mice 
were sacrificed at 10 days after inoculation. The lungs, kidneys and livers were aseptically removed and homogenized with a cell grinder in $3 \mathrm{ml}$ of sucrose phosphate glutamic acid buffer. Tissue suspensions were centrifuged at $500 \times \mathrm{g}$ for $10 \mathrm{~min}$ at $4^{\circ} \mathrm{C}$ to remove coarse tissue debris and were frozen at $-80^{\circ} \mathrm{C}$ until tested. For quantitation of MoPn in the homogenates of various organs, Hela 229 monolayer was inoculated with serially diluted tissue supernatants for $2 \mathrm{hr}$ and followed by culture for $36 \mathrm{hr}$. The cells having chlamydial inclusions were detected as those in the titration of purified EBs.

\section{Determination of MoPn-specific Antibody Levels}

Mice were sacrificed at 10 days postinfection. Mice sera were collected for the determination of MoPn-specific antibody (Ab) responses by using ELISA as previously described (27). Briefly, mice sera were added to 96-well plate coated with $10^{5}$ IFUs of MoPn organisms. Abs bound to the coated antigens were detected using biotin-conjugated anti-mouse immunoglobulin G (IgG)2a and IgGl (Caltag, Burlingame, CA) and followed by the addition of streptavidin and substrate (Sigma Inc., St. Louis, MI). Results were expressed as ELISA titers at $30 \mathrm{~min}$ using the endpoint [cutoff at optical density (O.D.) 405 $\mathrm{nm}, 0.5$ ] of the titration curves.

\section{Spleen Cell Culture and Cytokine Determination}

Spleens were collected at 10 days postinfection. Spleen cells were cultured at $7.5 \times 10^{6}$ cells $/ \mathrm{ml}$ with or without heat-killed EBs (HK-EBs; $5 \times$ $10^{4} \mathrm{IFU} / \mathrm{ml}$ ) in the presence or absence of 2 $\mathrm{ng} / \mathrm{ml}$ of rIL-12 (PharMingen, San Diego, CA), $20 \mathrm{ng} / \mathrm{ml}$ of rIL-18 (Research Diagnostic Inc., Flanders, NJ) or anti-mouse CD4 mAb (YTS191, kindly provided by Dr. Waldmann, University of Oxford, Oxford, U.K.) at $5 \mu \mathrm{g} / \mathrm{ml}$ for 3 days, as indicated. Culture supernatants were harvested for IFN- $\gamma$, IL-12, and IL-10 analysis by sandwich ELISAs. Ab pairs for determination of IFN- $\gamma$, IL-12, and IL-10 were purchased from PharMingen. Ab pairs for IL-18 were obtained from Research Diagnostic Inc. Cytokines in the lung homogenate supernatants were also tested using these ELISAs.

\section{Histopathological Analysis}

The lungs of MoPn-infected mice were isolated at day 10 postinfection and fixed in $10 \%$ buffered formalin and embedded in paraffin as previously described (6). Tissue sections (7 $\mu \mathrm{m})$ were then stained with hematoxylin and eosin and examined under light microscopy by a person blinded to the identification of mouse groups.

\section{Statistical Analysis}

Student's $t$-test was used for analysis of statistical significance ( $p$-value).

\section{Results}

Chlamydia Clearance in IL-18 KO and Caspase-1 KO Mice

To define the role of endogenous IL-18 on C. trachomatis MoPn lung infection, we examined in vivo chlamydial growth and disease severity in IL-18 KO mice following intranasal infection with a sublethal dose of MoPn. As shown in Fig. 1A, IL-18 KO mice showed slightly greater body weight loss than wild type (WT) mice, but the
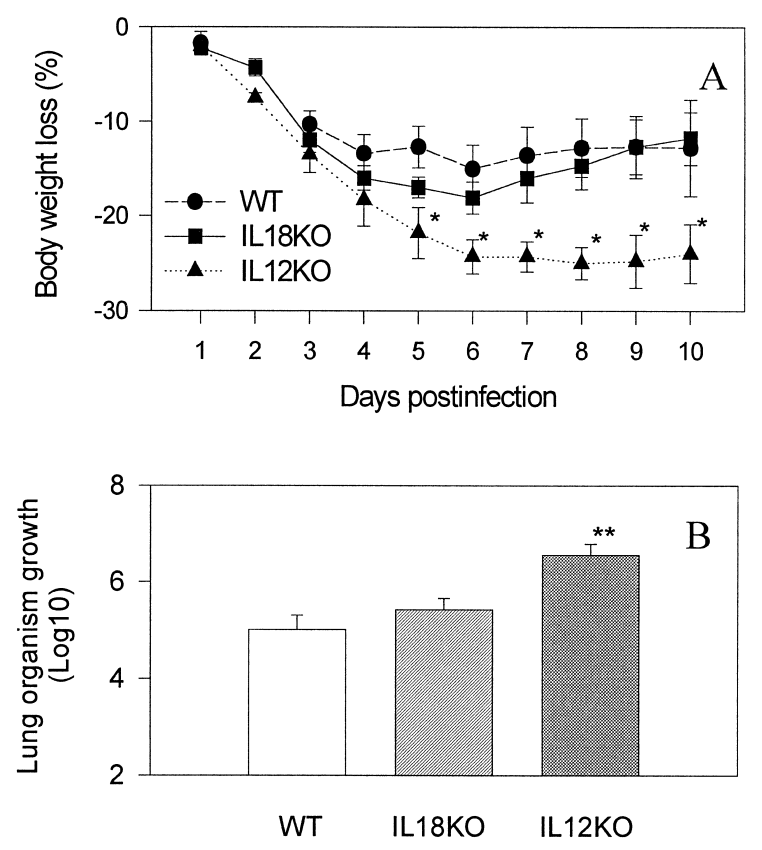

Fig. 1. Lung clearance and body weight changes after intranasal chlamydial infection. Three to four age- and sex-matched interleukin (IL)-18 knockout (KO), IL-12 KO and wild type (WT) mice were intranasally infected with $10^{4}$ inclusion forming units (IFU) of C. trachomatis mouse pneumonitis (MoPn) biovar and monitored daily for body weight changes. The mice were sacrificed at 10 days postinfection and the lung homogenates were analyzed for in vivo chlamydia growth as described in "Materials and Methods." The data represent the mean \pm standard error of the mean (SEM) of the percentages of body weight loss (A) or chlamydial IFU $\left(\log _{10}\right)$ per lung (B) from one of three separated experiments with similar results. ${ }^{*} p<0.05$ or ${ }^{* *} p<0.01$, compared with those in WT mice. 
recovery of lost body weight was similar for both groups of mice. IL-18 KO mice exhibited similar lung clearance as WT mice at 10 days following MoPn infection (Fig. 1B). Unlike IL-18 KO mice, IL-12 KO mice displayed severe disease with reduced ability to clear lung infection, as well as failure to recover lost body weight (Fig. 1A, B). Viable organisms were not recovered from livers and kidneys in all mouse groups when tested at day 10 following intranasal infection (data not shown), the time of peak in vivo chlamydial growth (7). This suggested that no significant dissemination of the infection had occurred in either IL-18 or IL-12 KO mice, at least during the early stages of infection. This finding was consistent with a previous report showing severe, but not disseminated, genital tract infection in IL-12-deleted mice (2). Since caspase- 1 is a cysteine protease that is required for the proteolytic activation of the IL-18 precursor into a bioactive form $(14,15)$, we took an alternative approach to evaluating the role of IL-18 in the development of anti-chlamydia immunity by using caspase-1 KO mice. Consistent with the observations in IL-18 KO mice, there was no significant difference in body weight loss (data not shown) and chlamydia growth in the lungs of caspase- $1 \mathrm{KO}$ and WT mice at 10 days following lung infection $[6.8 \pm 0.6(\mathrm{KO})$ vs. $6.3 \pm 0.5$ (WT), $\log _{10}$ IFU/lung, $\left.n=5, p>0.05\right]$. The results indicated that, unlike IL-12, endogenous IL-18 played a limited role in host defense against chlamydial infection.

\section{Immune Responses in IL-18 KO Mice}

\section{Following Chlamydia Lung Infection}

It has been established that $\mathrm{Th}_{1}$ cell-mediated immune responses play a dominant role in protection against chlamydial infection and that Thl or Th2 immune response can be inferred by the measurement of antibody isotypes and

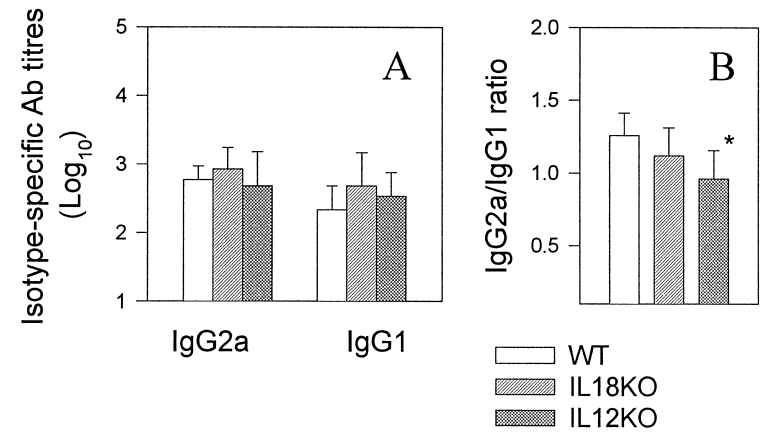

Fig. 2. MoPn-specific IgG2a and IgG1 responses following chlamydial infection. Age- and sexmatched interleukin (IL)-18 knockout (KO), IL-12 $\mathrm{KO}$ and wild type (WT) mice were intranasally infected with mouse pneumonitis $(\mathrm{MoPn})\left(1 \times 10^{4}\right.$ inclusion forming units (IFU)/mouse). Sera were collected at 10 days postinfection for determination of MoPn-specific immunoglobulin G (IgG)2a and IgGl using ELISA assays described in "Materials and Methods." The data are expressed as mean \pm standard error of the mean (SEM) of the titers $\left(\log _{10}\right.$ transformed) of all the sera in the same mouse group (A). The ratio of MoPn-specific IgG2a/IgGl is shown in (B). * $p<0.05$, compared with those in WT and IL-18 KO mice.

cytokine profile. We, therefore, examined the type of immune responses in $\mathrm{KO}$ and WT mice by measuring MoPn-specific serum IgG2a and IgGl Ab production and cytokine profiles after infection. Figure $2 \mathrm{~A}$ shows that there were no significant differences in $\mathrm{Ab}$ isotype levels among mouse groups tested. However, the ratio of MoPn-specific serum IgG2a/IgGl in IL-12 KO, but not in IL-18 KO mice, was significantly lower than those in WT mice postinfection (Fig. 2B), indicating impaired Thl immune responses in IL-12KO mice.

Th1 (IFN- $\gamma$ and IL-12) and Th2 (IL-10) cytokine production by spleen cells from infected IL-18 KO, IL-12 KO, and WT mice was also examined. As shown in Table 1, there were a

Table 1. Splenic interferon (IFN)- $\gamma$ production from mice intranasally infected with mouse pneumonitis (MoPn) $(\mathrm{u} / \mathrm{ml})^{a}$

\begin{tabular}{lccccc}
\hline & Medium & HK-EB & HK-EB + rIL-18 & HK-EB + rIL-12 & HK-EB + anti-CD4 \\
\hline Wild type & $<10$ & $426 \pm 85$ & $3075 \pm 1511$ & $3345 \pm 1511$ & $16 \pm 8$ \\
IL-18 KO & $<10$ & $139 \pm 32^{b}$ & $2014 \pm 446$ & $2116 \pm 742$ & $18 \pm 12$ \\
IL-12 KO & $<10$ & $16 \pm 6^{b}$ & $30 \pm 13^{b}$ & $2316 \pm 1142$ & $<10$
\end{tabular}

${ }^{a}$ Values are means \pm standard deviations from 4 to 9 mice each group at 10 days postinfection. ${ }^{b}$ Significantly different $(p<0.05)$, compared with those in wild type (WT) mice. HK-EB, heat-killed elementary bodies; recombinant interleukin (rIL); IL, interleukin; KO, knockout. 
moderate but significant decrease in the production of IFN- $\gamma$ following antigen specific restimulation of spleen cells from infected IL-18 $\mathrm{KO}$ mice. IFN- $\gamma$ production by antigen stimulated spleen cells was compromised much more severely in IL-12 KO mice, compared with IL$18 \mathrm{KO}$ and WT mice. Interestingly, although the addition of either exogenous IL-18 or IL-12 significantly enhanced IFN- $\gamma$ production in IL-18 KO mice, only IL-12, and not IL-18, significantly increased IFN- $\gamma$ production by spleen cells from IL-12 KO mice. There was no significant change in IL-12 or IL-10 production in IL-18 KO mice, and there was no significant change in IL-10 production in IL-12 $\mathrm{KO}$ mice (Fig. 3). CD4 T cells were the major source of IFN- $\gamma$ and IL-10 in the tested model system, because the addition of anti-CD4 mAb abolished most IFN- $\gamma$ and IL-10, but not IL-12, production (Table 1 and data not shown). The results indicated that, although both IL-12 and IL-18 play a role in Thl cell activation, exogenous IL-12 can replace the effect of IL-18, but not vice versa. This suggests that the critical role for IL-12 is in the initiation of Thl response following chlamydial infection.

\section{Pathological Changes and Local Cytokine Profiles in the Lungs of WT and KO Mice Following Chlamydia Infection}

We next analyzed the histopathological features and local cytokine profile in the lung after intranasal MoPn infection in WT and KO mice. The histological appearance of chlamydia-infected lung in WT mice was considerably different from that

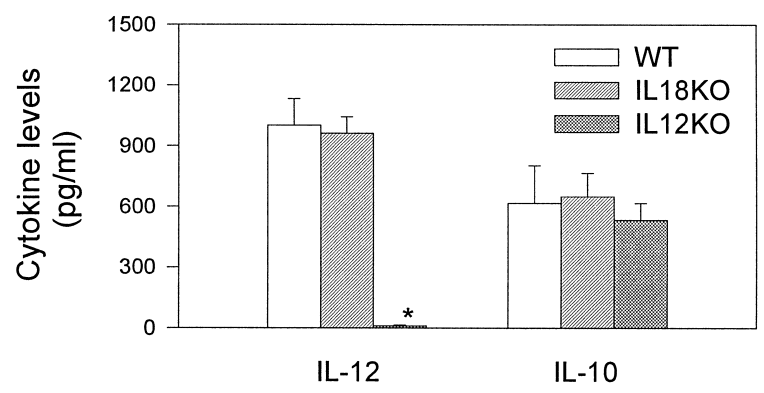

Fig. 3. Antigen-driven cytokine responses by spleen cells. Spleen cells from mouse pneumonitis (MoPn)-infected interleukin (IL)-18 knockout (KO), IL-12 KO and wild type (WT) mice were cultured at $7.5 \times 10^{6}$ cells $/ \mathrm{ml}$ with stimulation by heat-killed elementary bodies (EBs). Supernatants were harvested for IL-12 and IL-10 assays by ELISA. Data represented the mean \pm standard deviation (SD) of results from 4 to 9 mice each group after 3 days in culture. * represents undetectable level of IL-12. in either IL-18 KO or IL-12 KO mice (Fig. 4). The severity of inflammation and consolidation was more profound in $\mathrm{KO}$ mice, especially in IL-12 KO mice, compared with WT mice. The infiltrating cell types also appeared different among different groups of mice. In WT mice, the inflammatory infiltrates were composed of entirely mononuclear cells (Fig. 4C, D). In IL-18 KO mice, lung sections showed inflammation characterized by areas of consolidation with inflammatory exudate rich in both mononuclear cells and polymorphonuclear cells (Fig. 4E, F). Polymorphonuclear infiltrates were more numerous in IL-12 KO mice (Fig. 4G, H). We also measured local IFN- $\gamma$ production by testing lung homogenates (Table 2). Consistent with observations using spleen cell cultures, IFN- $\gamma$ levels were significantly lower in the lung homogenates of infected IL-18 KO and IL-12 KO mice than in WT mice.

\section{Discussion}

We characterized the in vivo role of endogenous IL-18 in the resolution of lung MoPn infection using gene knockout mice. The deficiency of IL- 18 was associated with a moderate reduction in systemic and lung IFN- $\gamma$ production, slightly more severe inflammatory reaction and body weight loss, but without measurably affecting MoPn lung clearance and the ratio of MoPn-specific IgG2a/Ig G1. Since IL-18 requires caspase- 1 activation for its biological function, we repeated these experiments using caspase- $1 \mathrm{KO}$ mice and, again, noted no significant differences in lung MoPn clearance between caspase-1 KO and WT mice at early stages of infection. In contrast, IL-12 KO mice showed significantly increased susceptibility to MoPn infection, characterized by severe body weight loss and organism growth in the lung, as well as impaired local and systemic IFN- $\gamma$ production and decreased MoPnspecific IgG2a/IgG1 ratio. The results suggest that, although both IL-18 and IL-12 are involved in the regulation of IFN- $\gamma$ production in vivo, IL-12, rather than IL-18, plays a more critical role in protective immunity against $C$. trachomatis MoPn lung infection.

Previous studies demonstrated that clearance of MoPn infection was exquisitely dependent on major histocompatibility complex (MHC) class II-mediated pathway, thus, indicating the critical role of $\mathrm{CD} 4 \mathrm{~T}$ cell-mediated 

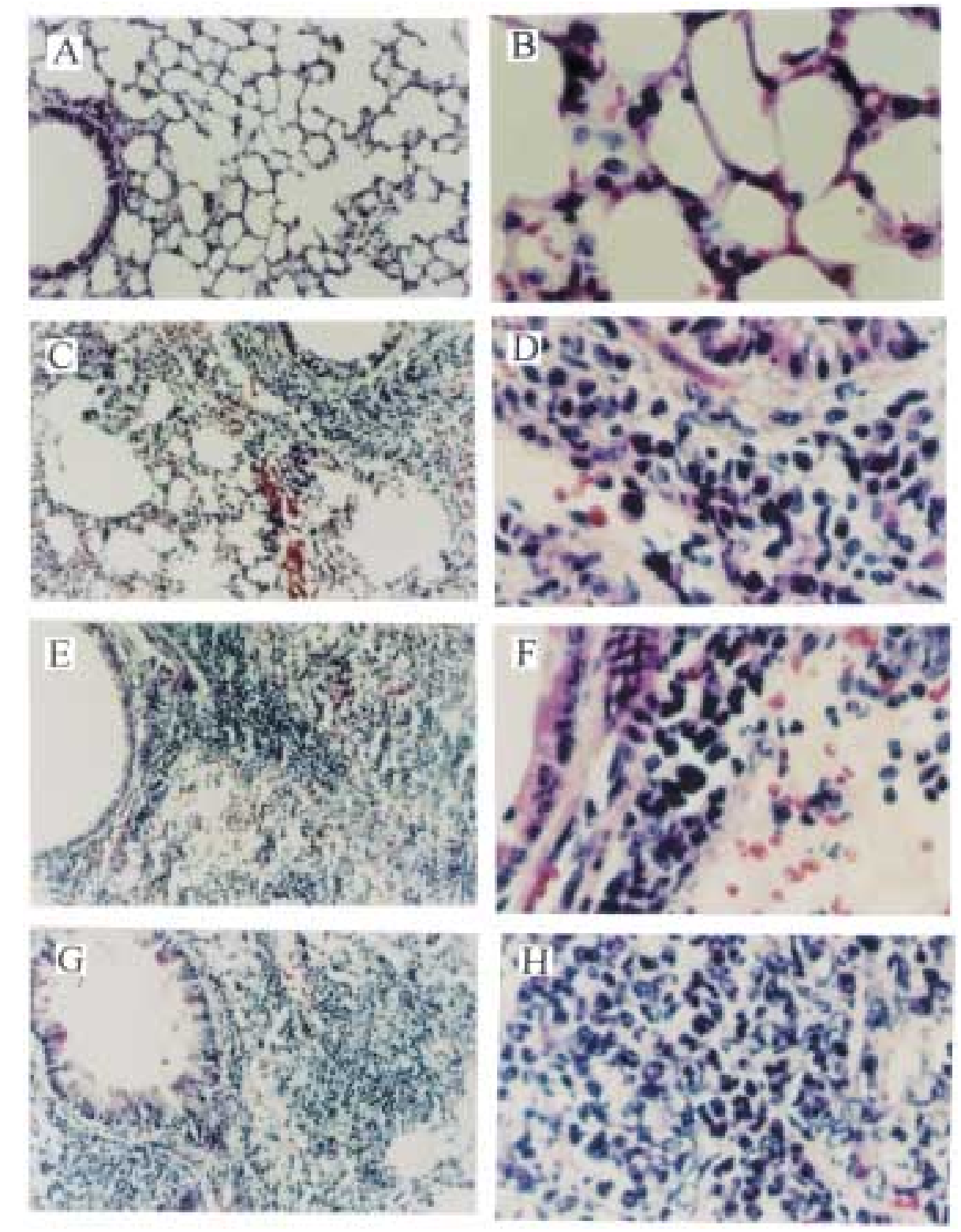

Fig. 4. Histological analysis of lung inflammatory reactions in mice following lung $C$. trachomatis mouse pneumonitis (MoPn) infection. Lung tissues were collected on day 10 postinfection and fixed by formalin and embedded in paraffin, followed by staining with hematoxylin and eosin. (A) and (B) show normal structure from naïve mice. Mice had bronchiolarinterstitial pneumonia with extensive infiltrates

immune responses in anti-chlamydial immunity $(1,4,5)$. The relative contribution of individual cytokines related to $\mathrm{CD} 4 \mathrm{~T}$-cell responses has been extensively studied in mouse models of chlamydial infection. In particular, recent studies suggest that IFN- $\gamma$, a typical Thl cytokine, is required for the prevention of systemic dissemination of MoPn infection following genital tract (2) and lung

mainly composed of mononuclear cells in wild type (WT) mice (C and D) and mixed polymorphonuclear and mononuclear cells in interleukin (IL)-18 knockout (KO) mice (E and F). IL-12 KO mice showed more severe inflammation with infiltration, predominately by polymorphonuclear cells $(\mathrm{G}$ and $\mathrm{H})$. Magnification, $200 \times(\mathrm{A}, \mathrm{C}, \mathrm{E}$ and $\mathrm{G}) ; 400 \times(\mathrm{B}, \mathrm{D}, \mathrm{F}$ and $\mathrm{H})$.

infection (6), as well as for resistance to rechallenge with $C$. trachomatis serovar D $(1,5)$. Johansson et al. $(1,5)$ compared the susceptibility to chlamydia infection of mice with either IFN- $\gamma$ receptor or IL-12 deficiency. They demonstrated that IFN- $\gamma$ receptor KO mice, but not IL-12 KO mice, remained susceptible to reinfection after recovered from primary infection although both kinds of KO mice 
Table 2. Cytokine levels in lung homogenates from mice intranasally infected with mouse pneumonitis (MoPn) ${ }^{a}$

\begin{tabular}{ccccc}
\hline & IFN $\gamma(\mathbf{u} / \mathbf{m l})$ & IL-12 $(\mathbf{p g} / \mathbf{m l})$ & IL-18 (pg/ml) & IL-10 (pg/ml) \\
\hline Wild type & $43 \pm 11$ & $3910 \pm 890$ & $3650 \pm 680$ & $415 \pm 41$ \\
IL-12KO & $13 \pm 1^{b}$ & ND $^{c}$ & $3300 \pm 810$ & $544 \pm 84$ \\
IL-18KO & $17 \pm 2^{b}$ & $3618 \pm 879$ & ND $^{c}$ & $433 \pm 37$ \\
\hline
\end{tabular}

${ }^{a}$ Values are means \pm standard deviations from 4 to 9 mice each group at day 10 postinfection. ${ }^{b}$ Significantly different $(p<0.05)$, compared with those in wild type (WT) mice. ' not detectable. IFN- $\gamma$, interferon- $\gamma$; IL, interleukin; KO, knockout.

showed significantly more severe primary genital tract infection, compared with WT mice. Thus, they concluded that IFN $-\gamma$ production by Thl cells was the particular important mechanism in immune protection against chlamydial infection.

Like IL-12, IL-18 has been shown to activate NK cells and induce IFN- $\gamma$ production by Thl cells. IL-18 has been reported to protect mice against pulmonary and systemic infection with Cryptococcus neoformans (28) and Yersinia enterocolitica (29), mainly through enhancing IFN- $\gamma$ production. IL-18 KO mice showed marked granulomatous lesions and increased mycobacterial growth in the lung, which was partially inhibited by the treatment with exogenous IL-18 (23). In the present study, the lung clearance of MoPn in IL-18 KO mice appears comparable to that in WT mice, although the former show relatively more severe signs of systemic illness, as manifested by slightly greater body weight loss and slightly more severe lung inflammatory reaction. Both IL-18 KO and WT mice recovered from primary infection and were highly resistant to secondary infection (data not shown). It has been reported that Thl and NK cells from IL-18 KO mice are still able to produce IFN- $\gamma$, although the levels are low (16). We also note less, but still significant, levels of IFN- $\gamma$ production in IL-18 KO mice following MoPn infection. It is possible, therefore, that the extent of IFN- $\gamma$ decrease in IL-18 KO mice is not severe enough to damage greatly the protective immunity to chlamydial infection. Another possibility is that MoPn can be partially cleared by IFN- $\gamma$-independent pathway, as suggested by Perry et al. (2), especially since IL-18 KO mice show normal levels of IL12 production, which may mediate protection via both IFN- $\gamma$ dependent and independent manners.

IL-18 and IL-12 can work synergistically in IFN- $\gamma$ production (21). IL-12 up-regulates the expression of IL- 18 receptor on cells producing IFN- $\gamma(30)$. It has been demonstrated that IL12-deleted mice or IL-12 KO mice display poor MoPn clearance and significantly reduced IFN$\gamma$ production following genital tract chlamydial infection $(1,2)$. Our studies using a lunginfected model shows that, although IL-12 can enhance IFN- $\gamma$ production in IL-18 KO mice, exogenous IL-18 fails to induce antigenspecific IFN- $\gamma$ production in MoPn-infected IL-12 KO mice. This suggests an essential role for IL-12 in Thl cell development following chlamydia infection. Collectively, the present and previous studies indicate that IL-12 is crucial for development of Thl response and MoPn clearance in both genital tract and lung infection models. The data showing that exogenous IL-12 greatly increases IFN- $\gamma$ production in both IL-18 KO and IL-12 KO mice suggest that IL-12 can also regulate IFN- $\gamma$ production through an IL-18 independent mechanism.

In conclusion, our study shows that, although both IL- 12 and IL-18 play a role in IFN- $\gamma$ production following lung chlamydial infection, IL-12 is the dominant cytokine regulating the Thl response that mediates protective immunity. The role of IL-18 is mainly ancillary and is not essential for organism clearance during chlamydial infection.

\section{Acknowledgments}

This work was supported by grants from the Medical Research Council of Canada and Aventis Pasteur.

\section{References}

1. Johansson M, Schon K, Ward M, Lycke N. (1997) Genital tract infection with Chlamydia trachomatis 
fails to induce protective immunity in gamma interferon receptor-deficient mice despite a strong local immunoglobulin A response. Infect. Immun. 65: 1032-1044.

2. Perry LL, Feilzer K, Caldwell HD. (1997) Immunity to Chlamydia trachomatis is mediated by $\mathrm{T}$ helper 1 cells through IFN- $\gamma$-dependent and independent pathways. J. Immunol. 158: 33443352.

3. Huang J, Wang MD, Lenz S, Gao D, Kaltenboeck B. (1999) IL-12 administered during Chlamydia psittaci lung infection in mice confers immediate and long-term protection and reduce macrophage inflammatory protein-2 level and neutrophil infiltration in lung tissue. J. Immunol. 162: 22172226.

4. Rottenberg ME, Gigliotti Rothfuchs AC, Gigliotti D, Svanholm C, Bandholtz L, Wigzell H. (1999) Role of innate and adaptive immunity in the outcome of primary infection with Chlamydia pneumoniae, as analyzed in genetically modified mice. J. Immunol. 162: 2829-2836.

5. Johansson M, Schon K, Ward M, Lycke N. (1997) Studies in knockout mice reveal that antichlamydial protection requires THI cells producing IFN- $\gamma$ : is this true for humans? Scand. J. Immunol. 46: 546-552.

6. Wang S, Fan Y, Brunham RC, Yang X. (1999) IFN- $\gamma$ knockout mice show Th2-associated delayed-type hypersensitivity and the inflammatory cells fail to localize and control chlamydial infection. Eur. J. Immunol. 29: 3782-3792.

7. Yang X, Gartner J, Zhu L, Wang S, Brunham RC. (1999) IL-10 gene knockout mice show enhanced Thl-like protective immunity and absent granuloma formation following Chlamydia trachomatis lung infection. J. Immunol. 162: 1010-1017.

8. Yang X, HayGlass KT, Brunham RC. (1996) Genetically determined differences in IL-10 and IFN- $\gamma$ responses correlate with clearance of Chlamydia trachomatis mouse pneumonitis infection. J. Immunol. 156: 4338-4344.

9. Igietseme JU, Ramsey KH, Magee DM, Williams DM, Kincy TJ, Rank RG. (1993) Resolution of murine chlamydial genital infection by the adoptive transfer of a biovar-specific, Thl lymphocyte clone. Reg. Immunol. 5: 317-324.

10. Puren AJ, Fantuzzi G, Gu Y, Su MS, Dinarello CA. (1998) Interleukin-18 (IFN $\gamma$-inducing factor) induces IL-8 and IL-1 $\beta$ via TNF $\alpha$ production from non-CD14+ human blood mononuclear cells. J. Clin. Invest. 101: 711-721.

11. Munder M, Mallo M, Eichmann K, Modolell M. (1998) Murine macrophages secrete interferon $\gamma$ upon combined stimulation with interleukin IL-12 and IL-18: a novel pathway of autocrine macrophage activation. J. Exp. Med. 187: 2103-2108.

12. Stoll S, Muller G, Kurimoto M, et al. (1997) Production of IL-18 (IFN- $\gamma$-inducing factor) mes- senger RNA and functional protein by murine keratinocytes. J. Immunol. 159: 298-302.

13. Stoll S, Jonuleit H, Schmitt E, et al. (1998) Production of functional IL-18 by different subtypes of murine and human dendritic cells (DC): DC-derived IL-18 enhances IL-12-dependent Thl development. Eur. J. Immunol. 28: 3231-3239.

14. Gu Y, Kuida K, Tsutsui H, et al. (1997) Activation of interferon- $\gamma$ inducing factor mediated by interleukin- $1 \beta$ converting enzyme. Science 275: 206-209.

15. Ghayur T, Banerjee S, Hugunin M, et al. (1997) Caspase- 1 processes IFN- $\gamma$-inducing factor and regulates LPS-induced IFN- $\gamma$ production. Nature 386: 619-623.

16. Takeda K, Tsutsui H, Yoshimoto T, et al. (1998) Defective NK cell activity and Thl response in IL-18-deficient mice. Immunity 8: 383-390.

17. Ohtsuki T, Micallef MJ, Kohno K, Tanimoto T, Ikeda M, Kurimoto M. (1997) Interleukin 18 enhances Fas ligand expression and induces apoptosis in Fas-expressing human myelomonocytic KG-1 cells. Anticancer Res. 17: 3253-3258.

18. Leite-De-Moraes MC, Hameg A, Arnould A, et al. (1999) A distinct IL-18-induced pathway to fully activate NK T lymphocytes independently from TCR engagement. J. Immunol. 163: 5871- 5876.

19. Hyodo Y, Matsui K, Hayashi N, et al. (1999) IL18 up-regulates perforin-mediated NK activity without increasing perforin messenger RNA expression by binding to constitutively expressed IL-18 receptor. J. Immunol. 162: 1662-1668.

20. Kohka H, Yoshino T, Iwagaki H, et al. (1998) Interleukin-18/interferon- $\gamma$-inducing factor, a novel cytokine, up- regulates ICAM-1 (CD54) expression in KG-1 cells. J. Leukoc. Biol. 64: 519- 527.

21. Dinarello CA. (1999) Interleukin-18. Methods 19: 121-132.

22. Dinarello CA, Novick D, Puren AJ, et al. (1998) Overview of interleukin-18: more than an interferon- $\gamma$ inducing factor. J. Leukoc. Biol. 63: 658664.

23. Sugawara I, Yamada H, Kaneko H, Mizuno S, Takeda K, Akira S. (1999) Role of interleukin-18 (IL-18) in mycobacterial infection in IL-18-genedisrupted mice. Infect. Immun. 67: 2585-2589.

24. Wei XQ, Leung BP, Niedbala W, et al. (1999) Altered immune responses and susceptibility to Leishmania major and Staphylococcus aureus infection in IL-18-deficient mice. J. Immunol. 163: 282 12828.

25. Mastroeni $P$, Clare $S$, Khan S, et al. (1999) Interleukin 18 contributes to host resistance and $\gamma$ interferon production in mice infected with virulent Salmonella typhimurium. Infect. Immun. 67: 478-483.

26. Fujioka N, Akazawa R, Ohashi K, Fujii M, Ikeda 
M, Kurimoto M. (1999) Interleukin-18 protects mice against acute herpes simplex virus type 1 infection. J. Virol. 73: 2401-2409.

27. Lu H, Zhong G. (1999) Interleukin-12 production is required for chlamydial antigen-pulsed dendritic cells to induce protection against live Chlamydia trachomatis infection. Infect. Immun. 67: 1763-1769.

28. Kawakami K, Qureshi MH, Zhang T, Okamura H, Kurimoto M, Saito A. (1997) IL-18 protects mice against pulmonary and disseminated infec- tion with Cryptococcus neoformans by inducing IFN- $\gamma$ production. J. Immunol. 159: 5528-5534.

29. Bohn E, Sing A, Zumbihl R, et al. (1998) IL-18 (IFN- $\gamma$-inducing factor) regulates early cytokine production in, and promotes resolution of, bacterial infection in mice. J. Immunol. 160: 299307.

30. Xu D, Chan WL, Leung BP, et al. (1998) Selective expression and functions of interleukin 18 receptor on $\mathrm{T}$ helper $(\mathrm{Th})$ type 1 but not Th2 cells. J. Exp. Med. 188: 1485-1492. 